\title{
DE MASCULINIDADES Y VIOLENCIA INSTITUCIONAL EN LA ORGANIZACIÓN ESCOLAR
}

ABOUT MASCULINITIES AND INSTITUTIONAL VIOLENCE IN SCHOOL ORGANIZATION

Jorge García Villanueva, Claudia Ivonne Hernández Ramírez

Universidad Pedagógica Nacional, México.

Correspondencia: jvillanueva@upn.mx

\section{RESUMEN}

En este trabajo se presentan los hallazgos preliminares de una investigación que examina las expresiones de masculinidad hegemónica en el ejercicio de actos de violencia institucional por parte de autoridades o grupos dominantes en una organización escolar de nivel superior. Los actos de violencia institucional se caracterizan por una aplicación parcial, discrecional, inequitativa, incluso ilegal, de las políticas y reglamentos institucionales. Como todo acto violento, la expresión institucional de éste va orientada a obligar a los otros al cumplimiento de la voluntad de quien ejerce el poder. Se describen las prácticas violentas que aplica un grupo dominante al interior de una universidad pública cuyos datos se reservan. Hasta el momento han sido reconocidos los siguientes elementos en el ejercicio de la violencia institucional por parte de este grupo: a) líderes ideológicos y morales, que son hombres, b) subjefes o líderes de segundo orden (donde figuran dos mujeres, una de ellas esposa de un líder ideológico), c) la comparsa, quienes son mujeres en su totalidad. Se identifican con claridad algunas expresiones asociadas con la masculinidad 
hegemónica, tales como: el ejercicio del poder reservado en los hombres, la posición jerárquicamente inferior por parte de las mujeres y su uso como agentes de mantenimiento del status quo.

Palabras clave: violencia institucional, masculinidad hegemónica, discriminación de género y organización escolar.

\section{ABSTRACT}

This paper presents the preliminary findings of an investigation that examines the expressions of hegemonic masculinity in the exercise of acts of institutional violence by authorities or dominant groups in a higher education organization. Acts of institutional violence are characterized by a partial, discretionary, inequitable, and even illegal application of institutional policies and regulations. As with any violent act, the institutional expression of this is aimed at forcing others to comply with the will of those who exercise power. We describe the violent practices applied by a dominant group within a public university whose data are confidential. So far, the following elements have been recognized in the exercise of institutional violence by this group: a) ideological and moral leaders, who are men, b) sub-chiefs or second order leaders (including two women, one of whom is the wife of an ideological leader), c) the comparsa, all of whom are women. Some expressions associated with hegemonic masculinity are clearly identified, such as: the exercise of power reserved for men, the hierarchically inferior position of women, and their use as agents for maintaining the status quo.

Key words: institutional violence, hegemonic masculinity, gender discrimination and school organization. 


\section{INTRODUCCIÓN}

La violencia ha sido un asunto de interés recurrente en las últimas décadas por parte de científicos sociales, particularmente, de la psicología social y, desde luego, de la antropología (Morales Domínguez, Moya Morales, Gaviria Stewart \& Cuadrado Guirado, 2007). Para efectos de este trabajo, se entenderá por violencia toda agresión encaminada a obligar a las personas a actuar en contra de su voluntad; mientras que la agresión se entiende, en términos de Morales Domínguez \& Moya Morales (1994), como un comportamiento hostil o destructivo (que no implica obligar al otro a hacer algo). Ante este panorama, se asume la noción de intencionalidad al definir la violencia, sin contradecir a quienes señalan ambos términos como sinónimos (lo cual es asunto de una amplia disertación).

Por otra parte, el estudio de la violencia institucional no parece tener tanta atracción para los investigadores sociales pues se encuentran muy pocas referencias a estudios relativos (Chavoya Peña, 2001). Este tipo de violencia tiene su legitimación social incluso en aspectos simbólicos, al igual que sucede con la violencia masculina (Ramírez Rodríguez, 2005) que está intrincada en muchos de los modelos identitarios de los hombres. Baste citar como ejemplo el uso de la fuerza pública por parte del Estado; nadie cuestiona que puede utilizarla en ciertos casos.

Hablar de violencia institucional es hacer referencia a un amplio conjunto de situaciones, que pueden llevarse a cabo desde las instituciones públicas a través de sus funcionarios que ejecutan a otras personas al violentar o atentar en contra de sus derechos humanos, al cometer abusos, actos ilícitos por medio de actos, acciones y omisiones (Ramírez Rodríguez, 2005). 
De acuerdo con Doz Costa (2010) existen formas de violencia institucional que surgen del mismo ordenamiento funcional del Estado; que se pueden naturalizar en la cultura institucional y ciudadana de una sociedad que con el tiempo pueden producir un modelo hegemónico de cultura política, en consecuencia, se presentan formas de violencia institucional, que refuerzan y propician círculos de marginalidad, exclusión y discriminación.

En este sentido, la hegemonía desde la perspectiva de Gramsci se puede entender "como el logro de un liderazgo moral, intelectual y político, a través de la expansión de un discurso que fija un significado parcial alrededor de puntos nodales. Involucra más que un consenso pasivo y acciones legítimas: envuelve la expansión de un particular discurso de normas, valores, puntos de vista y percepciones, a través de redescripciones persuasivas del mundo. La lógica de la hegemonía constituye una lógica de la articulación y de la contingencia” (Giacaglia, 2002, p. 155).

Hablar de violencia institucional denota un tipo de violencia que se gesta desde las instituciones formales del Estado o de sus ordenamientos disfrazados de mandatos de legitimidad que gozan del monopolio del uso de la fuerza y de la posibilidad de coaccionar y obligar en nombre de la ordenanza legal. Por tal razón, es necesario visibilizarla desde las estructuras institucionales y evitar la generación de obstáculos y así develar el aparato ideológico institucional que áun domina los espacios educativos (Doz Costa, 2010).

El objetivo de esta investigación fue identificar las expresiones de masculinidad hegemónica en el ejercicio de actos de violencia institucional por parte de autoridades o grupos dominantes en una organización escolar de nivel superior. 


\section{Masculinidad y violencia}

Especial interés han merecido los problemas de género relacionados con la violencia, en particular han surgido luminosos estudios acerca del ejercicio de la violencia por parte de los hombres, baste revisar trabajos de autores como Bourdieu (2005), Ramírez Rodríguez (2005), Ramírez Solórzano (2003). Se ha visto que la violencia suele ser componente de varias masculinidades, particularmente de la hegemónica, y han sido señalados sus estragos a lo largo de la historia de las relaciones entre los géneros.

Como lo han señalado Bourdieu (2005), Ramírez Solórzano (2003) y muchos autores más, la violencia implica la relación inequitativa entre los sujetos. En una relación violenta subyace la noción de que una de las personas es superior a la otra y, por tanto, puede ejercer poder sobre ésta última. En este sentido, el dominado puede o no estar de acuerdo con dicha relación, mientras que su contraparte se encargará de mantener su hegemonía. Este asunto ha sido estudiado en las relaciones hombre-mujer, en diferentes contextos (pareja, trabajo, familia) e, incluso, en las relaciones entre grupos de hombres donde algunos son considerados inferiores, según el contexto (heterosexual-homosexual, adulto-joven, adulto-viejo).

De acuerdo con Gonzáles Pagés y Fernández González (2009) la violencia es una de las problemáticas que ha afectado a las sociedades a través del tiempo con consecuencias graves y se ha convertido en el flagelo para las personas. Señalan que la violencia es el eje transversal de las relaciones sociales que se utiliza como un vehículo para la obtención y mantenimiento de un poder que convierte en sujetos dominantes a unos cuantos y subordina a la gran mayoría. Ejercer la violencia es una cualidad que se les impone a los hombres porque forma parte de la construcción de su masculinidad y sirve para perpetuar la posición privilegiada que tienen en el sistema, funge como una normativa imprescindible de cumplir. 
La violencia, identificada como parte sustancial de la masculinidad hegemónica, ha sido abordada en diversos estudios donde se muestran sus consecuencias en los procesos de formación de las identidades masculinas, en las relaciones de los hombres con las mujeres e, incluso, con otros hombres. Esto ha sido ilustrado en diversos trabajos como los de Renold (2003), Ramírez Solórzano (2003), Ramírez Rodríguez (2005), por citar algunos. Incluso se ha documentado la violencia masculina ejercida con la intención de forzar al otro a realizar actos que no necesariamente benefician a su dominador, como, por ejemplo; demostrar su heterosexualidad (Renold, 2003).

Para Kaufman (1989), los hombres construyen su identidad sobre los ejes de poder y dominio (lo que coincide con Bourdieu, 2005), lo que es, al mismo tiempo, una fuente de temor y dolor para ellos. Burin \& Meler (2004) comentan que el malestar de los hombres, expresado en la violencia que ejercen hacia los demás, radica en la construcción de su subjetividad (erigida en el poder y la violencia) y los estados de crisis derivados de la identificación con el género masculino (que los pone a prueba frecuentemente). No obstante, autores como Seidler (2006) apunta que las masculinidades están siendo transformadas por los propios hombres hacia modelos divergentes.

Por consiguiente, es indispensable repensar el rol que tienen los hombres en las sociedades porque en el proceso de construcción de la identidad masculina se privilegia la violencia como un medio para imponer su poder, pues las relaciones de género se construyen en relaciones de poder entre un sujeto masculino que es hegemónico y un sujeto femenino subordinado e invisibilizado (Cevasco, 2007). 
Según Vélez Bautista (2015) la masculinidad hegemónica vinculada al patriarcado, reproduce las desigualdades de género, entre ellas legítima la división sexual del trabajo presentándola como "natural" al igual que el ejercicio del poder. $\mathrm{Y}$ es desde la teoría de género que se pueden comprender las significaciones atribuidas al hecho de ser hombre o ser mujer en cada cultura y en cada sujeto y se visibiliza que las jerarquías no son naturales sino que han sido construidas mediante un proceso históricosocial en el que subyacen las relaciones de poder.

\section{Lo formal y lo informal en lo institucional}

Al referirse a la administración pública, autores como Scialpi (2005), Oszlak (1978) y Marmolejo, (2007) han señalado que la violencia institucional se manifiesta mediante el "imperialismo burocrático" donde se impone una modalidad de funcionamiento excluyente de grupos y organizaciones (dentro y fuera de la propia institución) que no la adopten. Desde esta perspectiva, la imposición administrativa no derivada de un proceso democrático genera exclusión de individuos y grupos e, incluso, de otras instituciones (Londoño, 2005).

Con el tiempo, la imposición normativa da lugar a que las organizaciones informales dentro de las instituciones de la administración pública desarrollen sus propias prácticas y las incorporen paulatinamente a la normatividad. En otras palabras, tras una imposición organizativa y normativa, las estructuras informales generan modificaciones a la norma hasta hacerla letra muerta o conseguir modificarla. Así, muchas de las prácticas institucionales son actos violentos legitimados, son parte de la cotidianidad de la vida en su interior (Cantón Mayo, 2003). 
En este trabajo se considera que la violencia institucional se manifiesta en actos que se caracterizan por una aplicación parcial, discrecional, inequitativa, incluso ilegal, de las políticas y reglamentos institucionales. Por ejemplo, la contratación laboral y autorización de viáticos para personas allegadas a quien autoriza dichos actos, la presión para obtener el voto en decisiones internas mediante el chantaje con beneficios otorgados, entre muchas otras prácticas.

De acuerdo con Bartlett (2014) entender las relaciones del trabajo como relaciones de poder implica verlas como relaciones asimétricas que tienden a producir formas particulares de comportamiento y representaciones, así como la generación de dispositivos que desarticulan maneras de organización colectiva. Es decir, formas de dominación que imponen sentidos y reglas que surgen de los conflictos cotidianos.

Lo interesante es comprender cómo un "campo estructurado de acción en el que individuos y grupos, dotados de medios distintos, situados en posiciones diferenciadas y movidos por objetivos no siempre convergentes o compatibles, e incluso en ocasiones contradictorios, establecen relaciones de colaboración, conflicto, negociación, y compromiso, no sólo sobre las modalidades operativas, sino sobre la definición de la finalidad misma de la organización, cuando ciertos grupos persiguen objetivos propios podrán o no generar modificaciones en la estructura interna. De haber cambios, pasado un tiempo en que la organización haya encontrado su equilibrio informal, tenderá a la materialización formal de este nuevo orden" (Crojethovic, 2012, p. 4). Además de entender que las masculinidades hegemónicas, al menos desde lo que explicita en este trabajo, tienden a involucrar patrones específicos de división interna y conflicto emocional, precisamente por su asociación con el poder de género (Connell \& Messerschmidt, 2005). 


\section{Contexto y estrategias de acercamiento}

El objetivo de esta investigación fue identificar las expresiones de masculinidad hegemónica en el ejercicio de actos de violencia institucional por parte de autoridades o grupos dominantes en una organización escolar de nivel superior. Se analizaron las experiencias reportadas por los informantes bajo las recomendaciones de Kvale \& Brinkmann (2009), es decir, desde el paradigma interpretativo porque se intentó comprender la realidad desde los significados de las personas en interacción mutua, también se pudo describir el hecho en el que se desarrolla el acontecimiento, esto es, la descripción contextual que posibilita la intersubjetividad en la captación de la realidad a través de una recogida sistemática de los datos que admite el análisis descriptivo (Ricoy Lorenzo, 2006).

La metodología de investigación fue cualitativa porque se orienta hacia la comprensión de las situaciones únicas y particulares, se centra en la búsqueda de significado y se interesa por la vivencia concreta en el contexto natural y en el contexto histórico de los agentes participantes, es decir, se interesa por la "realidad" tal y como la interpretan los sujetos, respetando el contexto donde dicha "realidad social" es construida (Rodríguez Gómez y Valldeoriola Roquet, 2009).

El estudio que se reporta se llevó a cabo al interior de una institución pública de educación superior en la Ciudad de México, a la que se denominará bajo el seudónimo Universidad Riquelme (UR), de la cual se omiten los datos de identificación a fin de proceder conforme los principios de ética profesional recomendados por la (International Union of Psychological Science, 2008). La UR cuenta con más de tres décadas de existencia. Dadas las dimensiones de la UR, se tomó como muestra una de sus divisiones más representativas por el número de estudiantes (casi 1000) y profesores adscritos (poco más de 80). 
Sin generalizar a todas sus divisiones, en la que fue objeto de este estudio, existe un grupo dominante claramente identificado (líderes ideológicos) formado por cinco hombres. En el siguiente nivel, se encuentran los líderes de segundo orden, que los representan dos hombres y dos mujeres que son reconocidos como parte del grupo dominante, pero no como sus principales líderes. En el tercer nivel de esta organización informal se encuentra el grupo al que se designó como la comparsa, formada por, al menos nueve mujeres y ningún hombre.

Existe un grupo de profesores por contrato temporal (interinatos) en el que hay tres hombres (entre 35 y 40 años) y una mujer. Este grupo no es considerado parte de la comparsa del grupo dominante porque cuentan con su propia líder moral que es, a la vez, opositora al grupo dominante. Asimismo, el resto de los docentes se organiza en otros grupos de trabajo no vinculados con el dominante pero pertenecientes a la misma división.

La información recabada para este trabajo proviene de entrevistas individuales realizadas a diversos miembros de la comunidad universitaria: 3 profesoras, 2 profesores, 2 estudiantes (un hombre y una mujer); en la tabla 1 se muestran algunas de sus características, las consideradas relevantes para el estudio que se reporta. Los criterios de selección de los informantes fueron: tener más de 3 años de antigüedad en la institución, no formar parte de los líderes del grupo dominante y, en el caso de los estudiantes, haber realizado el servicio social dentro del área de estudio. 
Tabla 1

Características de las personas informantes

\begin{tabular}{|c|c|c|c|c|c|c|}
\hline Informante & Edad & $\begin{array}{c}\text { Status } \\
\text { de pareja }\end{array}$ & Alma máter & Posgrado & Antigüedad & Contrato* \\
\hline Profesora 1 & 40 & Unión libre & UR & Maestría & 10 años & $\begin{array}{c}\text { Tiempo completo, } \\
\text { definitivo }\end{array}$ \\
\hline Profesora 2 & 36 & Casada & UR & Maestría & 3 años & $\begin{array}{c}\text { Tiempo completo, } \\
\text { interinato }\end{array}$ \\
\hline Profesora 3 & 34 & Soltera & Otra & No & 4 años & $\begin{array}{l}\text { Tiempo completo, } \\
\text { interinato }\end{array}$ \\
\hline Profesor 1 & 44 & Casado & Otra & Doctorado & 10 años & $\begin{array}{c}\text { Tiempo completo, } \\
\text { definitivo }\end{array}$ \\
\hline Profesor 2 & 35 & Soltero & Otra & Maestría & 4 años & $\begin{array}{c}\text { Tiempo completo, } \\
\text { interinato }\end{array}$ \\
\hline Alumno & 25 & Unión libre & UR & -- & 5 años & Egresado \\
\hline Alumna & 25 & Soltera & UR & -- & 5 años & Egresada \\
\hline
\end{tabular}

${ }^{*} \mathrm{El}$ contrato por interinato requiere su renovación semestral o anual y se asigna discrecionalmente por parte de la autoridad (jefe inmediato y jefe de personal). La definitividad o contrato por tiempo ilimitado se obtiene por concurso de oposición abierto.

\section{RESULTADOS PRELIMINARES}

Hasta el momento han sido reconocidos los siguientes actores en el ejercicio de la violencia institucional por parte del grupo dominante:

a) líderes ideológicos y morales, que son hombres,

b) subjefes o líderes de segundo orden (donde figuran dos mujeres, una de ellas esposa de un líder ideológico), y

c) la comparsa, quienes son mujeres en su totalidad.

Se identifican con claridad algunas expresiones asociadas con la masculinidad hegemónica, tales como: el ejercicio del poder reservado en los hombres, la posición jerárquicamente inferior por parte de las mujeres y su uso como agentes de mantenimiento del status quo. A continuación se explican estos aspectos.

Los líderes y subjefes son hombres en su gran mayoría (sólo figuran dos mujeres en el segundo nivel), de los cuales son dos quienes ostentan la autoridad actualmente (uno de ellos es jefe de la división). Todas las integrantes de la comparsa están o han estado vinculadas con los líderes 
bajo uno de estos roles: alumnas, parejas o esposas. A la vez, ellas tienen en promedio, menor grado académico que los líderes (licenciatura, la mayor parte de ellas, tres con maestría y una con doctorado). Una de estas profesoras coordina un área de importancia en la división.

Primero fui su alumna [del actual jefe de la división] en la licenciatura, luego hice la maestría y mientras me invitó a dar clases aquí. Ya luego hubo concurso y gané mi plaza... (Profesora 1, 40 años).

En este punto se observa claramente, en términos de Bourdieu (2005) la dominación masculina ejercida en las mujeres. Si bien podría aplaudirse su contratación por ser mayoría de mujeres con empleo, el trasfondo de esto implica razones políticas y no de cambio social. Cabe señalar que la apertura de concursos de oposición no depende del jefe de la división sino de la máxima autoridad.

Salvo cuatro profesoras, todas las demás que integran la comparsa son contratadas por tiempo limitado (interinato), lo cual implica cierta obediencia al grupo dominante y le garantiza a éste un número de votos a favor cuando se requiere de tomar decisiones "democráticas". Existe pues una difusa coerción permanente en este grupo de mujeres que le "deben" a los líderes su contratación. La frase de la profesora 2 ilustra lo anterior:

Yo estoy muy agradecida con él [el actual jefe de la división] porque primero fui su alumna y ahora me ha dado la oportunidad de ser maestra aquí. En un gran logro para mi ser maestra de la universidad donde estudié y trabajar con un maestro tan bueno como él (Profesora 2, 36 años). 
A decir del profesor 1, es muy claro que "meten a trabajar a sus mujeres porque las controlan". En este sentido, el informante señala que ellas son simples repetidoras del discurso de los líderes o bien, ejecutan sin más las acciones que se les indiquen.

Para los miembros de otros grupos de la misma división, el grupo dominante "se reparte los recursos de los viáticos y a los demás casi no nos toca nada" (Profesor 1, 44 años). La profesora 3 añade: "ya sabes... tienes que estar con ellos para que te toque [autorización de viáticos u otros beneficios económicos]". Desde estos argumentos, se observa que el ejercicio del presupuesto es poco equitativo, a pesar de que en el grupo dominante "no se realizan proyectos de investigación que generen publicaciones arbitradas ni apoyo a tesistas" (Profesor 2, 35 años).

Incluso los estudiantes informantes se percatan de esta problemática y señalan:

Bueno, Elvira hizo su servicio social con él y vela, ahora es becaria y hasta lo cubre en las clases. Al rato, va a ser maestra. Lo mismo pasa con Rebeca, por eso está muy pegada a la coordinadora, a ver qué le saca (Alumno 1, 25 años).

No entiendo cómo meten a cualquiera a dar clases. Está bien que hayan sido sus alumnas, pero se pasan. Deben seleccionarlas mejor... Da coraje ver que además son muy pasivas, sumisas... Les falta experiencia, preparación (Alumna 1, 25 años).

Como puede denotarse, estos estudiantes conocen de la situación porque realizaron el servicio social dentro de la división y pudieron observar y 
escuchar a los profesores y sus comentarios sobre la vida institucional. Como ninguno de los dos fue pupilo del jefe de la división, no se benefician de la beca que ahora tiene su compañera que sí laboró con él. Además, el alumno 1, por ser hombre, ya quedaría fuera del apoyo que suele brindar el grupo dominante.

En resumen, existe un machismo que se disfraza de apoyo a las mujeres pero que, en realidad, es una forma de control laboral sobre ellas. Se les ve como elementos de segundo orden, votos seguros y apoyo en mano de obra para los intereses del grupo dominante. Todo esto, con el consentimiento expreso de estas mujeres, lo cual hace aún más invisible la violencia de género que impera en la relación.

Por otra parte, los miembros de otros grupos distintos al dominante se ven discriminados en la asignación de recursos para su operación. Y los alumnos que no se relacionan con el jefe de la división y los otros líderes también se ven discriminados al no acceder a los beneficios económicos que dan a sus allegados. Es necesario comprender que las relaciones del trabajo son relaciones de poder que están atravesadas por relaciones asimétricas $\mathrm{y}$ que tienden a producir formas particulares de comportamiento $\mathrm{y}$ representaciones, a su vez, implican grados de legitimación y "concesión" por las partes, sea en forma directiva, valor, norma u ordenanza (Bartlett, 2014).

Es importante comprender que el acercamiento a esta organización formal es pieza clave para vislumbrar la calca de una organización informal patriarcal que manifiesta cómo son las relaciones al interior de ésta y que son parte de la academia de esta institución educativa. Para esto resulta necesario efectuar estudios sociométricos en las universidades públicas para evidenciar la apropiación de los claustros por parte de grupos informales, que ejercen el machismo desde un sistema que se reviste de ideologías y prácticas perniciosas como la dominación patriarcal. 


\section{REFERENCIAS}

Bartlett, J. (2014). Estudios críticos de administración: dominación simbólica y relaciones de poder. Theomai, 30, 8-15.

Bourdieu, P. (2005). La dominación masculina. Barcelona: Anagrama.

Burin, M. y Meler, I. (2004). Varones. Género y subjetividad masculina. Buenos Aires: Paidós.

Cantón Mayo, I. (2003). La estructura de las organizaciones educativas y sus múltiples implicaciones. Revista Interuniversitaria de Formación del Profesorado, 17(2), 139-165.

Cevasco, G. (2007). Masculinidad/es y violencia. Chacarera, 34, 47-50.

Connell, R. \& J. Messerschmidt (2005). Hegemonic Masculinity: Rethinking the concept. Gender \& Society, 19(6), 829-859.

Crojethovic, M. (2012). El aspecto informal de las organizaciones públicas: instrumento de dominación y estrategia para el cambio. Cuadernos del CENDES, 29(79), 1-19.

Chavoya Peña, L. (2001). Organización del trabajo y culturas académicas. Estudio de dos grupos de investigadores de la Universidad de Guadalajara. Revista Mexicana de Investigación Educativa, 6(11), 1-12.

Doz Costa, J. (2010). Violencia institucional y cultura política Cuadernos de la Facultad de Humanidades y Ciencias Sociales- Universidad Nacional de Jujuy, 38, 145-168.

Giacaglia, M. (2002). Hegemonía. Concepto clave para pensar la política. Tópicos, 10,151-159.

Gonzáles Pagés, J. y Fernández González, D. (2009). Masculinidad y violencia: aproximaciones desde el universo del deporte. Educar, Curitiba, 35, 123-136.

International Union of Psychological Science. (2008). Universal Declaration of Ethical Principles for Psychologists. Recuperado el 22 de 01 de 2010, 
de Psychology Around the World Home Page: http://www.iupsys.net/ index.php/ethics/declaration

Kaufman, M. (1989). Hombres. Placer, poder y cambio. Santo Domingo: CIPAF.

Kvale, S. \& Brinkmann, S. (2009). InterViews. Learning the Craft of Qualitative Research Interviewing (Segunda edición ed.). Londres: Sage.

Londoño, F. (2005). Un análisis sobre la dinámica de los grupos de investigación en Colombia: de su conformación a su supervivencia. Investigación \& Desarrollo, 13(1), 184-203.

Marmolejo, D. (julio de 2007). La violencia institucional. Movimiento. Revista del Movimiento Ciudadano Metropolitano, 2-6.

Morales Domínguez, J. F. y Moya Morales, M. C. (1994). Agresión. En J. F. Morales Domínguez, M. C. Moya Morales, E. Rebolloso, J. M. Fernández-Dols, C. Huici y J. Marques. Psicología Social (pp. 465491). Madrid: McGraw Hill.

Morales Domínguez, J. F., Moya Morales, M. C., Gaviria Stewart, E. y Cuadrado Guirado, I. (2007). Psicología Social. Madrid, España: McGraw Hill.

Oszlak, O. (1978). Políticas públicas y regímenes políticos: reflexiones a partir de algunas experiencias latinoamericanas. Estudios Cedes, 3(2), 44-76.

Ramírez Rodríguez, J. C. (2005). Madejas entreveradas. Violencia, masculinidad y poder. México: Plaza y Valdés.

Ramírez Solórzano, M. A. (2003). Hombres violentos. Un estudio antropológico de la violencia masculina. México: Plaza y Valdés.

Renold, E. (2003). 'If You Don't Kiss Me, You're Dumped': Boys, boyfriends and heterosexualised masculinities in the primary school. Educational Review, 55(2), 179-194.

Ricoy Lorenzo, C. (2006). Contribución sobre los paradigmas de investigación. Educação. Revista do Centro de Educação, 31(1), 11-22. 
Rodríguez Gómez, D. y Valldeoriola Roquet, J. (2009). Metodología de la investigación. Barcelona: Universidad Oberta de Catalunya.

Scialpi, D. (2005). Violencia laboral y desamparo institucional aprendido. Jurisprudencia argentina, MOBBING: El acoso psicológico en el ámbito laboral, 1-24.

Seidler, V. J. (2006). Transforming masculinities. Men, cultures, bodies, power, sex and love. Nueva York: Routledge.

Vélez Bautista, G. (2015). Masculinidades. Poder, identidad y violencia de género. En G. Vélez Bautista y A. Luna Martínez. (eds.). Violencia de género. Escenarios y quehaceres pendientes. (pp.233-254). Estado de México: Universidad Autónoma del Estado de México.

Envío a dictamen: 16 de marzo de 2021

Reenvío: 25 de mayo de 2021

Aprobación: 17 de junio de 2021

Jorge García Villanueva. Psicólogo. Doctor en Psicología por la Universidad Nacional Autónoma de México (UNAM). Profesor titular en la Universidad Pedagógica Nacional (UPN) y miembro de la Academia Mexicana de Ciencias (AMC). Su publicación más reciente es el libro La identidad masculina en jóvenes: una mirada, México, UPN, 2017, 172 pp. (Versión gratuita en inglés descargable: A Look into Masculine Identity in Mexican Young Men). Correo electrónico: jvillanueva@upn.mx

Claudia Ivonne Hernández Ramírez. Licenciada en Psicología Educativa, Especialista de Género en Educación y Maestra en Desarrollo Educativo perteneciente a la línea de investigación Educación en Ciencias, por parte de la UPN. Actualmente, trabaja en una investigación que está focalizada en el estudio de las diferentes formas de ser hombre. Colabora como asistente de investigación en temas de género en educación en su Alma Mater. Correo electrónico: cihernandez@upn.mx 